\title{
The significance of biological, environmental, and social risk factors for prostate cancer in a cohort study in Brazil
}

Frederico R. Romero, Antonio W. Romero, Rui Manuel S. de Almeida, Fernando Cesar de Oliveira Jr., Renato Tambara Filho

Hospital Policlínica Cascavel (FRR, AWR), Faculdade Assis Gurgacz (FRR, RMSA), Cascavel, Instituto Curitiba de Saúde (FRR, FCOjr), Curitiba and Hospital de Clínicas da Universidade Federal do Paraná (FRR, RTF), Curitiba, PR, Brazil

\section{ABSTRACT}

Purpose: To evaluate the significance of several risk factors for prostate cancer in a cohort of Brazilian men.

Subjects and methods: Men $\geq 40$ years-old participating in a prostate cancer screening program between December 2006 and April 2011 in the city of Curitiba, Brazil, were evaluated to determine the prevalence, relative risk (RR) and 95\% CI of prostate cancer according to age, race, ethnicity, family history of prostate cancer, educational level, and history of vasectomy, increased blood pressure, diabetes mellitus, and urethritis.

Results: In 2121 men included in this study, prostate cancer prevalence was $0.6 \%$ for men between 40-49 years versus 2.0\% (adjusted $R R=2.58$ ), 7.7\% (adjusted $R R=5.76$ ), and 8.4\% (adjusted $R R=4.88$ ) for men 50-59 years, 60-69 years, and $\geq 70$ years, respectively ( $\mathrm{p}<0.05$ to all). The prevalence of cancer was $5.1 \%$ in blacks versus $3.3 \%$ in whites (adjusted $\mathrm{RR}=1.56, \mathrm{p}>0.05)$; 6.1\% in African descendants, in comparison to 3.0\% in non-African descendants (adjusted RR $=3.17, \mathrm{p}<0.05$ ); 5.1\% in men with a positive family history, compared to $2.5 \%$ in those with no family history (adjusted RR $=1.55, \mathrm{p}>0.05)$; and $4.8 \%$ in participants with incomplete elementary school level or lower, compared to $2.2 \%$ in men with complete elementary school level or higher education (adjusted $R R=1.85, \mathrm{p}>0.05$ ). Men with/without history of vasectomy, increased blood pressure, diabetes, and urethritis had a prostate cancer prevalence of $0.8 \% / 3.0 \%$ (adjusted RR = 0.23, p > 0.05), 3.8\%/2.2\% (adjusted RR = 1.16, p > 0.05), 3.7\%/2.6\% (adjusted $R R=1.39, p>0.05$ ), and 2.6\%/2.6\% (adjusted $R R=0.99, p>0.05$ ), respectively. Conclusions: Risk factors associated with an increased prevalence of prostate cancer in this cohort included increasing age and African ethnicity.

\section{ARTICLE INFO}

\section{Key words:}

Epidemiology; Population

groups; Prevalence;

Prostatic Neoplasms

Int Braz J Urol. 2012; 38: 769-78

Submitted for publication:

March 12, 2012

Accepted after revision:

October 10, 2012

\section{INTRODUCTION}

Prostate cancer is one of the most common visceral malignant neoplasms in men worldwide. The clinical incidence and mortality of prostate cancer vary widely between different geographic regions, with the lowest rates in Asia, and the highest in North America and Scandinavia $(1,2)$.

Although the specific causes of prostate cancer are not completely known, epidemiological evidence suggests that biological, environmental, and social risk factors play a role in the initiation and progression of this disease $(1,2)$. 
Differences in exposure to these factors among various populations may be responsible for geographic variations in prostate cancer rates. Studies of the potential risk factors associated with prostate cancer are important to identify the significance of each factor in specific populations for counseling, to determine entry into screening programs, and as a means of comparison between different regions or countries.

Little has been published about the risk factors for prostate cancer in the Brazilian population. In this prospective study, we evaluated the importance of several acknowledged risk factors for prostate cancer in a cohort of Brazilian men.

\section{MATERIALS AND METHODS}

All men attending a prostate cancer education program conducted in the city of Curitiba (PR) as part of the city employees' Health Care System were invited to join this survey. Inclusion criteria were men $\geq 40$ years of age willing to undergo prostate cancer screening. The study protocol was reviewed and approved by the Institutional Ethics Committee on Human Research (registry number 2253.147/2010-06).

Subjects accepting to participate this study were interviewed and examined by a single Urologist. Participants were recommended to follow-up annually, and data was collected prospectively between December 2006 and April 2011.

Clinical evaluation consisted of complete medical history, digital rectal examination (DRE), and serum PSA determination. Participants with prostate nodularity suspicious for cancer, and/or a PSA level $\geq 4.0 \mathrm{ng} / \mathrm{mL}$ were indicated for ultrasound-guided 12-core prostate biopsy. We excluded subjects with prior history of prostate cancer, those who did not return to follow-up, and those that elected not to undergo biopsy.

Outcomes of interest included prevalence, relative risk (RR) and 95\% confidence intervals (95\% CI) of prostate cancer according to age (5059 versus $40-49,60-69$ versus $40-49$, or $\geq 70$ versus 40-49 years), race (black versus white, or brown versus white), ethnicity (African descendants versus non-African descendants), family history of prostate cancer (yes versus no), education (in- complete elementary school level or lower versus complete elementary school level or higher), and personal history of vasectomy, increased blood pressure, diabetes mellitus, and sexually transmitted urethritis (yes versus no, to all). Univariate (non-adjusted) analysis was calculated using the Fisher's Exact Test or Pearson's Chi-square, whichever appropriate. Multivariate (adjusted) analysis was performed using logistic regression models (IBM SPSS Statistics, version 20.0.0). Statistical significance was set when $p<0.05$ or when the 95\%CI did not include the null hypothesis $(95 \% \mathrm{CI}$ $\neq 1.00)$.

\section{RESULTS}

From 2499 men initially enrolled in this cohort, we excluded 323 participants that did not return to follow-up, and 22 men with history of prostate cancer. Indications for prostate biopsy were present in $244(11.3 \%$, t. 2154) subjects, of whom 33 (13.5\%, t. 244) elected not to undergo biopsy and were also excluded. Finally, 2121 men with a mean follow-up of 21.5 (range 1 to 52) months were included in the analysis (Table-1), 58 of whom (27.5\% of all biopsies [t. 211], and 2.7\% of all participants [t. 2121]) were diagnosed with prostate cancer.

\section{Age}

Participants with 40-49 years, 50-59 years, 60-69 years, and $\geq 70$ years had a prevalence of prostate cancer of, respectively, 0.6\%, 2.0\%, $7.7 \%$, and $8.4 \%$. Compared to men aged between 40-49 years, the prevalence of prostate cancer was 3.2 times higher in those 50-59 years (non-adjusted RR 3.19, $\mathrm{p}=0.009$; adjusted RR 2.58, $\mathrm{p}=0.049), 12.4$ times increased for those 60-69 years (non-adjusted RR 12.42, p < 0.001; adjusted RR 5.76, p < 0.001), and 13.5 times greater for those $\geq 70$ years-old (non-adjusted RR 13.54, p < 0.001; adjusted RR 4.88, $\mathrm{p}=0.019$ ) (Table-2).

\section{Race}

The prevalence of prostate cancer in black participants was 5.1\% (non-adjusted RR 1.56, p $=0.082$; adjusted RR 1.56, $\mathrm{p}=0.599$ ), and it was $2.6 \%$ in brown individuals (non-adjusted RR 0.80, 
$\mathrm{p}=0.479$; adjusted RR 0.75, $\mathrm{p}=0.427$ ), compared to $3.3 \%$ in white men (Table-2).

\section{Ethnicity}

The prevalence of cancer in African descendants was $6.1 \%$, in comparison with $3.0 \%$ of that in non-African descendants (non-adjusted RR 2.06, $\mathrm{p}=0.070$; adjusted RR 3.17, $\mathrm{p}=0.024)(\mathrm{Ta}-$ ble-2).

\section{Family history}

The prevalence of prostate cancer in participants with a positive family history was 5.1\%, in contrast to $2.5 \%$ of those with negative family history (non-adjusted RR 2.01, p = 0.036; adjusted RR 1.55, $\mathrm{p}=0.289$ ) (Table-2).

\section{Education}

The prevalence of prostate cancer in participants with incomplete elementary school level or lower was $4.8 \%$, compared to $2.2 \%$ in men with complete elementary school level or higher education (non-adjusted RR 2.17, $\mathrm{p}=0.004$; adjusted RR 1.85, $\mathrm{p}=0.062$ ) (Table-2).

\section{Vasectomy}

In the group of participants with prior history of vasectomy, $0.8 \%$ was diagnosed with prostate cancer, in comparison to 3.0\% of those with no history of vasectomy (non-adjusted RR $0.26, p=0.016$; adjusted RR 0.23, p = 0.150) (Table-2). Most individuals with a positive history of vasectomy were aged $<60$ years $(90.7 \%$, n. 235/t. 259). In 137 (52.9\%, t. 235) men aged 40-49 years, the mean interval between vasectomy and screening was of $8.1( \pm 6.0)$ years. The 98 (37.8\%, t. 235) participants $50-59$ years had a mean interval between vasectomy and screening of $13.2( \pm 6.8)$ years. In the $24(9.2 \%$, t. 235$)$ men $\geq 60$ years of age, the mean interval between vasectomy and screening was $17.6( \pm 6.5)$ years.

\section{Increased blood pressure}

The prevalence of prostate cancer in men with arterial hypertension was 3.8\%, compared with $2.2 \%$ of those with no history of hypertension (non-adjusted RR 1.74, $\mathrm{p}=0.031$; adjusted RR 1.16, $p=0.677$ ) (Table-2).
Diabetes mellitus

Between participants with self-reported diabetes, the prevalence of prostate cancer was $3.7 \%$, compared with $2.6 \%$ of men with no history of diabetes (non-adjusted RR 1.39, p = 0.108; adjusted RR 1.39, $\mathrm{p}=0.563$ ) (Table-2).

\section{Sexually transmitted urethritis}

Prostate cancer was diagnosed in 2.6\% of men with a history of urethritis, and in $2.6 \%$ of those with no history of disease (non-adjusted RR $1.02, p=0.959$; adjusted RR 0.99, $\mathrm{p}=0.904)(\mathrm{Ta}-$ ble-2).

\section{DISCUSSION}

Prostate cancer has been known as a disease of the elderly men (2). Age is known as the most significant risk factor for prostate cancer $(1,2)$. Diagnosis is rare before the age of 50 but, after this age, incidence and mortality both increase almost exponentially (2). In this study, overall prostate cancer prevalence increased from approximately 1:200 (0.6\%) men 40-49 years, and 1:50 (2.0\%) men 50-59 years, to 1:13 (7.7\%) men 60-69 years, and 1:12 (8.4\%) men $\geq 70$ years of age.

Black men have the highest reported incidence of prostate cancer in the world, with an increased risk in several populations including North American, Caribbean, Scandinavian, and Britain (3-7). Although the risk of prostate cancer in the present study was not significantly increased for black versus white men (adjusted RR 1.56, 95\%CI 0.64-3.82), similarly to several other Brazilian studies (8-11), a meta-analysis evaluating the prevalence of prostate cancer in black versus white men in Brazil, including the results of our study, demonstrated that the pooled risk of prostate cancer was significantly increased in blacks (RR 1.55, 95\%CI 1.32-1.82), suggesting that race is a significant risk factor for prostate cancer in Brazilian men, and that the individual results of most published studies may have been limited by a low sample, a reduced proportion of black men, or a small rate of prostate cancer in each study group (12).

Stratified by ethnicity, African descendants showed a significantly increased prevalence of prostate cancer compared to non-African 


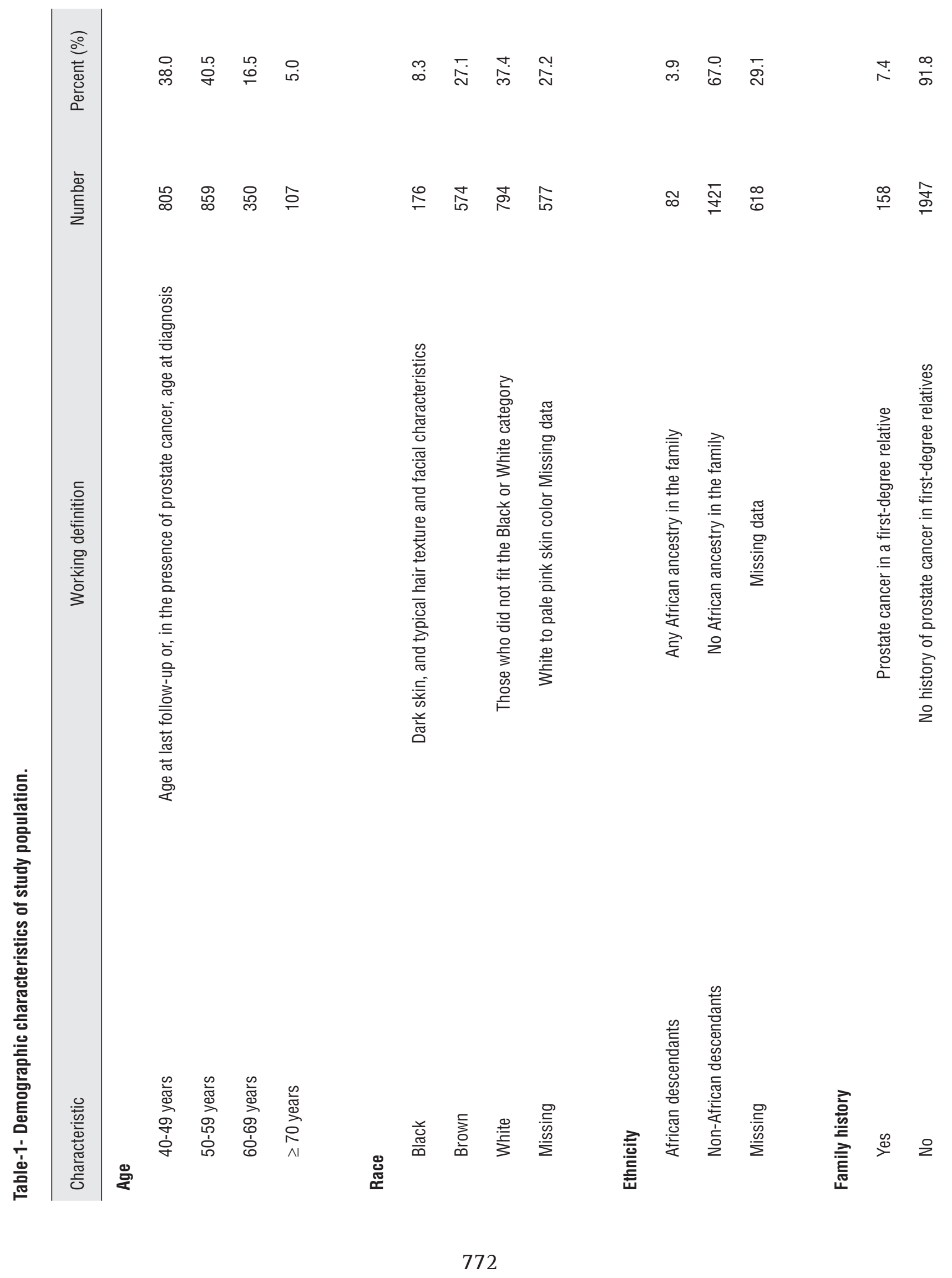




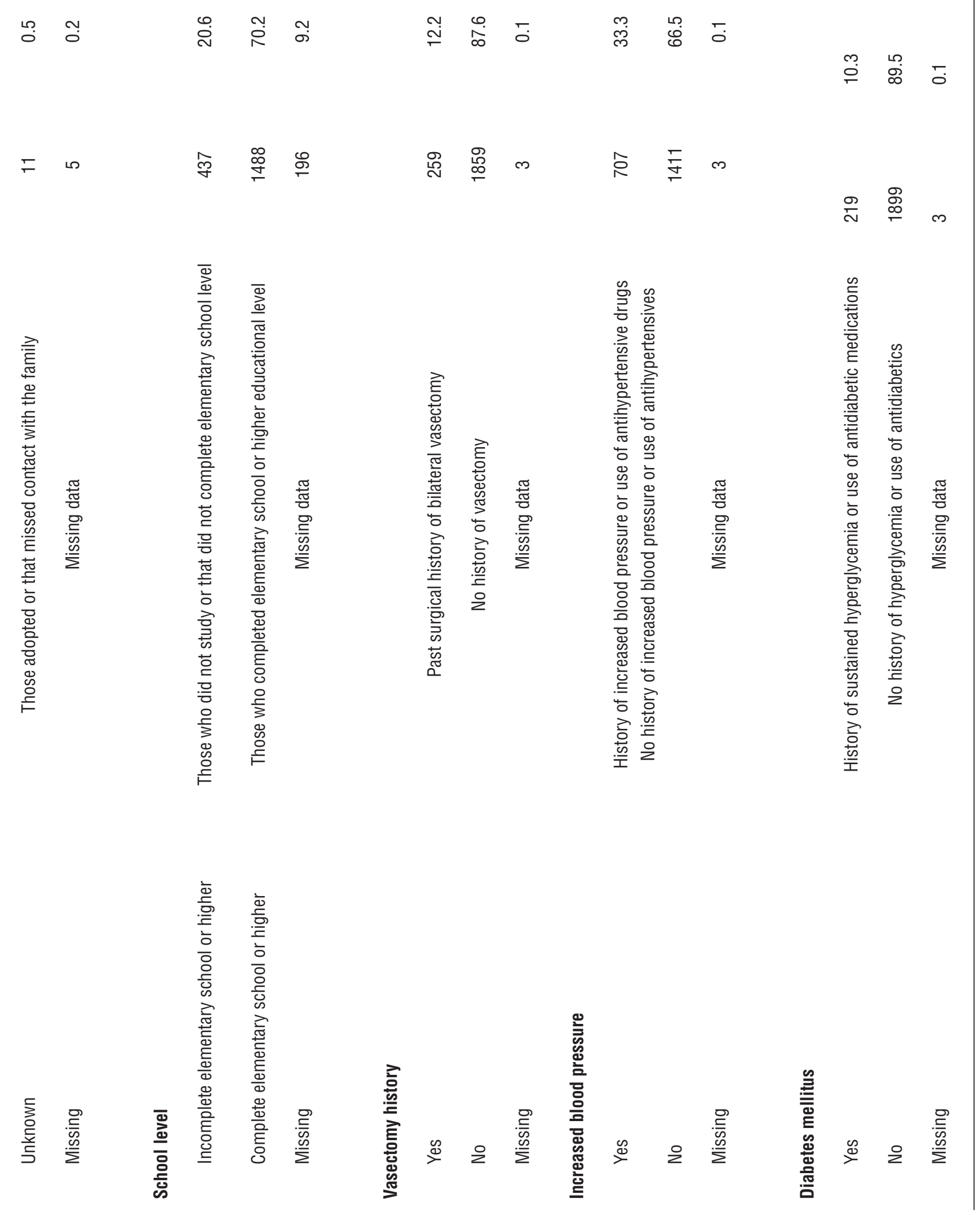




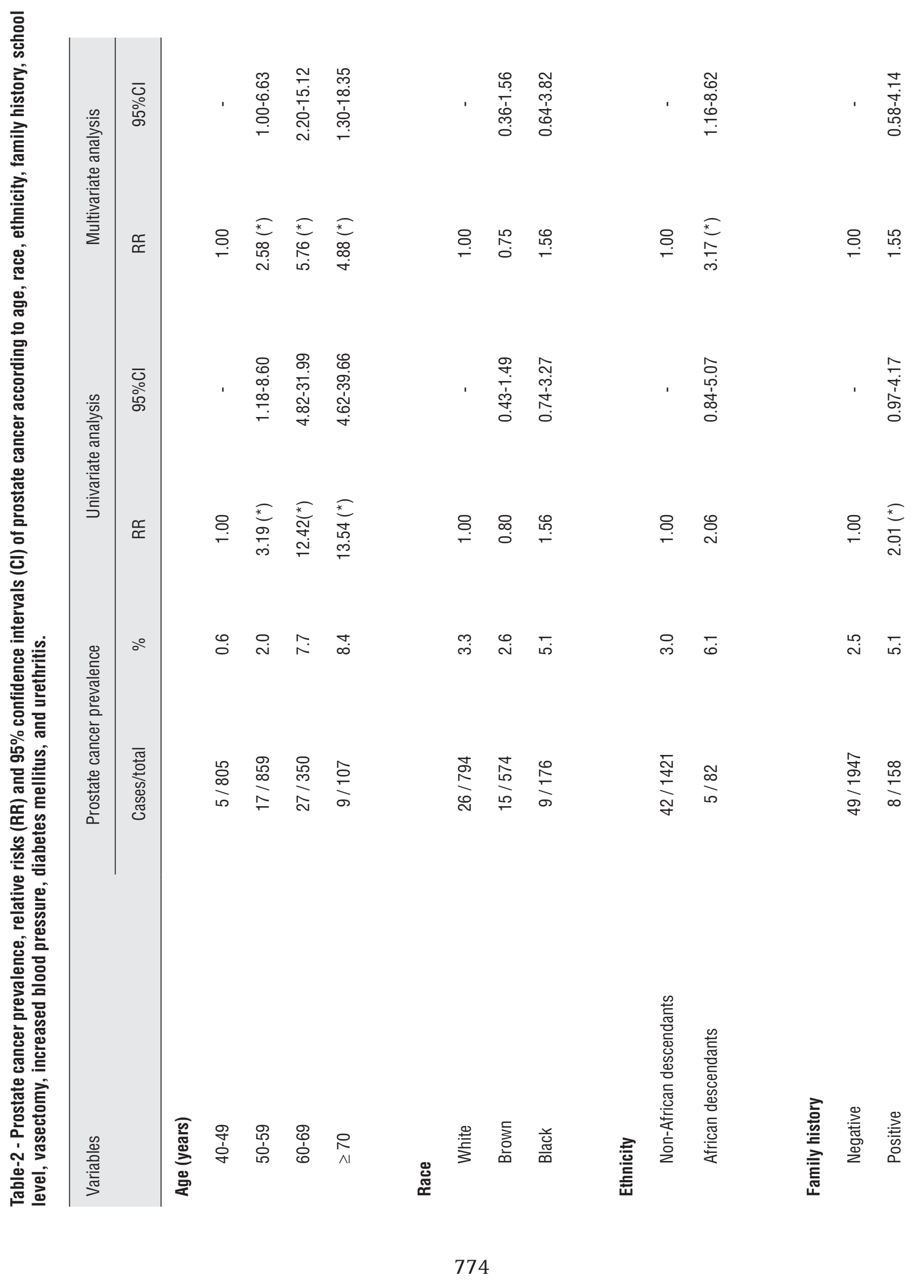




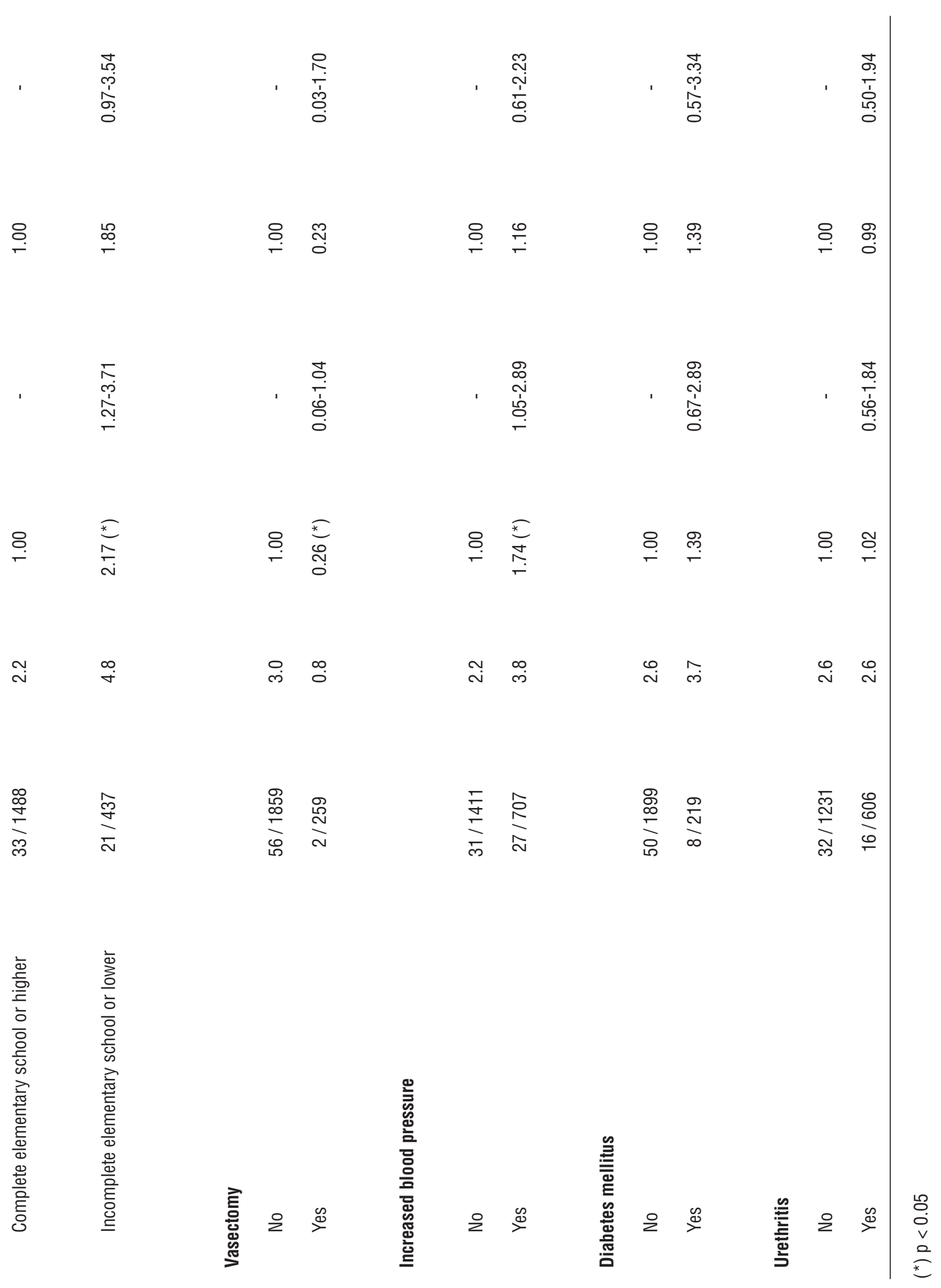


descendants in multivariate analysis (adjusted RR 3.17, 95\%CI 1.16-8.62). This result is comparable to the only other Brazilian survey that used African ancestry as a criteria to classify men in different racial/ethnic groups while evaluating the risk of prostate cancer (13).

Ample epidemiologic evidence suggests that prostate cancer has both a familial and genetic component (1). In one meta-analysis, the pooled risk of prostate cancer was higher in men who had a father or brother affected by prostate cancer, compared to a control population with no affected relatives (RR 2.5, 95\%CI 2.2-2.8) (14). Another meta-analysis showed an increased risk of prostate cancer for any affected family member (RR 2.04, 95\%CI 1.64-2.55), a higher risk for disease for affected first-degree relatives (RR 2.24, 95\%CI 2.08-2.41), and an elevated risk of cancer in affected second-degree relatives (RR 1.91, 95\%CI 1.58-2.30) (15). In the present research, although there was a marginal increased prevalence of prostate cancer in men with affected first-degree relatives in univariate analysis (non-adjusted RR 2.01, 95\%CI 0.97-4.17, $\mathrm{p}<0.05$ ), the risk of cancer became similar between men with and those without family history of prostate cancer in multivariate regression (adjusted RR 1.55, 95\%CI $0.58-4.14)$. These results may have been limited by the lack of knowledge or forgetfulness of the diagnosis of prostate cancer in a family member (measurement bias), or by unknown intervening factors (confounding bias).

Existing data suggest that the incidence of different types of cancer, including that of the prostate, increases with decreasing socioeconomic status (2). Socioeconomic factors may influence the risk of developing prostate cancer indirectly, influencing dietary factors, occupational exposure, access to health care, the type of care available, and even the attitudes and concerns over health matters exhibited by the various populations (confounding bias) $(2,16)$. Our data showed that men with incomplete elementary school or lower school level had a higher risk of prostate cancer compared to those with complete elementary school or higher education (non-adjusted RR 2.17, 95\%CI 1.27-3.71). However, since lower school levels were more prevalent in participants $\geq 60$ years $(41.4 \%)$, compared to those $<60$ years of age (17.9\%), as well as in black (31.4\%) versus white individuals $(20.4 \%)$, both of whom have a potentially increased risk of prostate cancer, we must acknowledge that the association between cancer of the prostate and lower education may be biased by age, race, or other unidentified confounding variables. The absence of statistically significant difference in multivariate analysis (adjusted RR 1.85, 95\%CI 0.97-3.54) supports this hypothesis.

Several studies have associated a history of vasectomy with an increased risk for prostate cancer, although an equal number of studies provide evidence to the contrary (2). One meta-analysis reported an elevated risk of prostate cancer after vasectomy (RR 1.37, 95\%CI 1.15-1.62), with a linear trend suggesting a 10\% increase for each additional 10 years since vasectomy up to 30 years (RR 1.32, 95\%CI 1.17-1.50) (17). Nevertheless, the results of two other meta-analyses showed no difference in the risk of prostate cancer between subjects with and without a history of vasectomy, regardless of the time since surgery $(18,19)$. Our study demonstrated an inverse relation between vasectomy and prostate cancer (non-adjusted RR 0.26, 95\%CI 0.06-1.04, $\mathrm{p}<0.05)$. However, most participants with a history of vasectomy were 4049 years-old, suggesting that the inverse association may be the result of a lower prevalence of prostate cancer in the younger men most frequently undergoing vasectomy (confounding bias), as well as a shorter time distance since vasectomy, not long enough for prostate cancer to develop (susceptibility bias). The inverse association between vasectomy and prostate cancer may also be influenced by detection bias. It is possible that men undergoing vasectomy were more likely to be examined with serum PSA level or DRE, which led to the earlier discovery of prevalent prostate cancers, resulting in a spuriously reduced occurrence of prostate cancer in later years.

The association between blood pressure and prostate cancer has been poorly investigated. In a prospective study, Martin et al. found that hypertension was associated with an increased risk in developing prostate cancer (RR 1.05, 95\%CI 1.01-1.10) (20). A small case-control study in Bra- 
zil also showed a significantly greater occurrence of arterial hypertension in men with prostate cancer (21). Our findings demonstrated an increased univariate risk of prostate cancer in men with history of increased blood pressure (non-adjusted RR 1.74, 95\%CI 1.05-2.89). However, given the frequent occurrence of these conditions in ageing men, a large proportion of patients can be expected to suffer from such an association (confounding bias), which may explain the loss of statistical significance in multivariate analysis (adjusted RR 1.16, 95\%CI 0.61-2.23).

A lower risk of prostate cancer among diabetics has been suggested by several studies, including two separate meta-analysis $(22,23)$. Although not significant, our results showed an overall increased risk of 39\% in the risk of prostate cancer between diabetics and nondiabetics (adjusted RR 1.39 95\%CI 0.57-3.34). Potential confounding factors (such as diet, body weight, and physical activity), inaccuracies in the diagnosis of diabetes (measurement bias), and failure to account for time since the diagnosis (susceptibility bias) may have limited our findings. Furthermore, age-adjusted risk of prostate cancer in diabetics > 60 years-old, allegedly with a longer time of disease, was decreased in comparison with men in the same age group without diabetes (non-adjusted RR 0.86, 95\%CI 0.37-2.01), potentially supporting an inverse association between prostate cancer and long time-history of diabetes.

Chronic inflammation leading to cellular hyperproliferation to replace damaged tissue contributes to the development of infection-associated cancers of the colon, esophagus, stomach, bladder, and liver. Accumulating epidemiologic, histologic, and genetic evidences suggest that a similar process may underlie the development of prostate cancer $(1,16,24)$. Notwithstanding, the results of our study demonstrated no association between a past history of urethritis and the development of prostate cancer. A potential source of bias in studies evaluating self-reported history of sexually transmitted diseases (STDs) is measurement bias, given the difficulty in identifying oligosymptomatic infections or undiagnosed outbreaks of the disease (under diagnosis bias), and the possible acknowledgement of other urologi- cal conditions, such as balanoposthitis or urinary tract infections, as if they were STDs (overdiagnosis bias). Furthermore, given the social connotations of STDs, patients may not be forthcoming about their history of disease (social convenience bias) or may not recall being diagnosed with STDs (recall bias).

Besides the limitations described specifically for each potential risk factor, another shortcoming of our study is the relatively small sample of participants $\geq 70$ years, given that the study population was built mainly of active municipal employees from an established private Health Care System. The subjects in this study also had a potentially lower prevalence of black race, as well as an increased percentage of participants with higher educational levels, compared to the overall population. Notwithstanding, the frequency of hypertension and diabetes in our sample was similar to the estimated prevalence of hypertension and diabetes in the population of the same age living in the city of Curitiba (25), which partially validates our findings.

\section{CONCLUSIONS}

The risk factors associated with an increased prevalence of prostate cancer, in our sample of Brazilian men, include increasing age, and African ethnicity.

\section{ABBREVIATIONS}

$$
\begin{aligned}
& \text { PSA = Prostate Specific Antigen } \\
& \text { RR = Relative Risk } \\
& \text { 95\%CI = 95\% Confidence Interval } \\
& \text { STDs = Sexually transmitted diseases }
\end{aligned}
$$

\section{ACKNOLEDGEMENTS}

This article is part of a doctoral thesis in development by the main author at the Surgery Department of Federal University of Paraná, Brazil.

\section{CONFLICT OF INTEREST}

None declared. 


\section{REFERENCES}

1. Klein EA, Platz EA, Thompson IM: Epidemiology, etiology, and prevention of prostate cancer. In: Wein AJ, Kavoussi LR, Novick AC, Partin AW, Peters CA (ed.), Campbell-Walsh urology, 9th ed. Philadelphia, Saunders Elsevier. 2007; pp. 2854-7.

2. Haas GP, Sakr WA: Epidemiology of prostate cancer. CA Cancer J Clin. 1997; 47: 273-87.

3. Miller BA, Kolonel LN, Bernstein L, Young JL, Swanson GM, West DW, et al.: Racial/ethnic patterns of cancer in the United States 1988-1992. Bethesda, MD: National Cancer Institute. 1996; pp. 96-4104.

4. National Cancer Institute: Genetics of prostate cancer Available at: URL: http://www.cancer.gov/cancertopics/ pdq/genetics/prostate/HealthProfessional\#Section_ Last accessed: September 18, 2012.

5. Bouchardy C, Mirra AP, Khlat M, Parkin DM, de Souza JM, Gotlieb SL: Ethnicity and cancer risk in São Paulo, Brazil. Cancer Epidemiol Biomarkers Prev. 1991; 1: 21-7.

6. Ben-Shlomo Y, Evans S, Patel B, Anson K, Muir G, Persad $R$, et al.: Differences in the epidemiology and presentation of prostate cancer in Black and White men in England: lessons learnt from the process study. BJU Int. 2009; 103: 723-4.

7. Jack RH, Davies EA, Møller H: Prostate cancer incidence, stage at diagnosis, treatment and survival in ethnic groups in South-East England. BJU Int. 2010; 105: 1226-30.

8. Martins AC, Reis RB, Suaid HJ, Maciel LM, Cologna AJ, Falconi RA: Screening for carcinoma of the prostate in volunteers. Int Braz J Urol. 2000; 26:516-22.

9. Antonopoulos IM, Pompeo AC, Hayek OR, Sarkis AS, Alfer Jr W, Arap S: Results of prostate cancer screening in nonsymptomatic men. Int Braz J Urol. 2001; 27:227-34.

10. Glina S, Toscano IL Jr, Mello JF, Martins FG, Vieira VL, Damas CG: Results of screening for prostate cancer in a community hospital. Int Braz J Urol. 2001; 27:235-43.

11. Barros MS, Silva VR, Santos GB, Hughes A, Silveira MA: Prevalence of prostate adenocarcinoma according to race in an university hospital. Int Braz J Urol. 2003; 29: 306-11; discussion 312.

12. Romero FR, Romero AW, de Almeida RM, Tambara Filho $R$ : The prevalence of prostate cancer in Brazil is higher in Black men than in White men: systematic review and metaanalysis. Int Braz J Urol. 2012; 38: 440-7.

13. Paschoalin EL, Martins AC, Pastorello M, Sândis KA, Maciel LM, Silva WA Jr, et al.: Racial influence on the prevalence of prostate carcinoma in Brazilian volunteers. Int Braz J Urol. 2003; 29: 300-5.

14. Johns LE, Houlston RS: A systematic review and metaanalysis of familial prostate cancer risk. BJU Int. 2003; 91: 789-94.
15. Bruner DW, Moore D, Parlanti A, Dorgan J, Engstrom P: Relative risk of prostate cancer for men with affected relatives: systematic review and meta-analysis. Int $\mathrm{J}$ Cancer. 2003; 107: 797-803.

16. Ferrís-I-Tortajada J, Berbel-Tornero 0, Garcia-I-Castell J, López-Andreu JA, Sobrino-Najul E, Ortega-García JÁ: Non dietetic environmental risk factors in prostate cancer. Actas Urol Esp. 2011; 35: 289-95.

17. Dennis LK, Dawson DV, Resnick MI: Vasectomy and the risk of prostate cancer: a meta-analysis examining vasectomy status, age at vasectomy, and time since vasectomy. Prostate Cancer Prostatic Dis. 2002; 5: 193-203.

18. Tang LF, Jiang H, Shang XJ, Zhao LM, Bai Q, Hong K, et al.: Vasectomy not associated with prostate cancer: a metaanalysis. Zhonghua Nan Ke Xue. 2009; 15: 545-50.

19. Sharlip ID: Vasectomy: American Urological Association (AUA) Guideline. Linthicum: AUA Foundation, 2012. Available at: URL: http://www.auanet.org/content/media/vasectomy.pdf. Last accessed: September 18, 2012.

20. Martin RM, Vatten L, Gunnell D, Romundstad P: Blood pressure and risk of prostate cancer: Cohort Norway (CONOR). Cancer Causes Control. 2010; 21: 463-72.

21. de Santana IA, Moura GS, Vieira NF, Cipolotti R: Metabolic syndrome in patients with prostate cancer. Sao Paulo Med J. 2008; 126: 274-8.

22. Kasper JS, Giovannucci E: A meta-analysis of diabetes mellitus and the risk of prostate cancer. Cancer Epidemiol Biomarkers Prev. 2006; 15: 2056-62

23. Bonovas S, Filioussi K, Tsantes A: Diabetes mellitus and risk of prostate cancer: a meta-analysis. Diabetologia. 2004; 47: 1071-8.

24. Taylor ML, Mainous AG 3rd, Wells BJ: Prostate cancer and sexually transmitted diseases: a meta-analysis. Fam Med. 2005; 37: 506-12.

25. Brazil, Ministry of Health, National Cancer Institute: Coordination of prevention and surveillance. Household survey on risk behaviors and morbidity of diseases and nontrasmissible diseases: Brazil, 15 capital and Federal District, 20022003. Rio de Janeiro: INCA, 2004. Available at: URL: http:// www.inca.gov.br/inquerito/docs/completa.pdf. Last accessed: September 18, 2012.

Correspondence address: Dr. Frederico R. Romero R. Mato Grosso, 1979, Centro Cascavel, PR, 85812-025, Brazil Fax: + 5545 3225-3003 E-mail: frederico.romero@gmail.com 